\title{
DOENÇA DE HALLERVORDEN-SPATZ
}

\author{
DESCRIÇÃO DE UM CASO
}

\author{
J. ADAMO Jr.*
}

\begin{abstract}
RESUMO - relatado o caso de paciente do sexo masculino de 19 anos de idade, com quadro progressivo de distúrbio de comportamento e đe postura distônica, com início nas extremidades do hemicorpo direito há 5 anos. Ao exame apresenta síndrome extrapiramidal e piramidal, sendo o diagnóstico de doença de Hallervorden-Spatz confirmado pela ressonância nuclear magnética do cranio (RM). O objetivo deste registro é ressaltar la importâncta da RM no estudo de doenças dugenerativas do sistema nervoso.
\end{abstract}

PALAVRAS-CHAVE: distonia, doença degenerativa, doença de Hallervorden-Spatz, ressonância nuclear magnética.

\section{Hallervorden-Spatz disease: a case report.}

SUMMARY - It is reported the case of a male patient with 19 years old presenting progressive behavior disturbance and dystonic posture beginning on right, with approximately five years of evolution. Extrapyramidal and pyramidal syndrome was evidenced at examination. This finding suggests Hallervorden-Spatz disease which was confirmed by resonance imaging (MRI). The purpose of reporting this case is to call attention for the importance of MRI in the study of degenrative diseases of the nervous system.

KEY WORDS: dystonia, degenerative disease, Hallervorden-Spatz disease, magnetic resonance imaging.

Sabemos que a doença de Hallervorden-Spatz é de natureza heredodegenerativa autossômica recessiva e que seus primeiros sinais e sintomas surgem nas duas primeiras décadas da vida. O diagnóstico definitivo, antes da ressonância nuclear magnética (RM) somente era possível ao exame anátomo-patológico.

Neste registro é relatado o caso de paciente com doença de HallervordenSpatz, cujo diagnóstico foi confirmado por RM.

O objetivo do registro deste caso é ressaltar a importância da RM na doença de Hallervorden-Spatz.

\section{RELATO DO CASO}

WEA, paciente do sexo masculino, com 19 anos de idade. Há 7 anos apresenta agreswiv1dade e nervosismo, bem como abuso de álcool, maconha e cocaína que parou há 3 anos. Há 5 anos associou-se ao quadro dificuldade para deambular e para abrir a mão direita; há 1 ano, dificuldade para fechar a boca e para movimentar o hemicorpo esquerdo, sendo notada rouquidăo, com disfagia, períodos de dispnéia e movimentos bruscos de flexão e torção dos membros superiores e pescoço. Nos antecedentes familiares refere primo materno com quadro seme-

*Médico Assistente, Clínica Neurológica, Hospital do Servidor Público Municipal de São Paulo. Aceite: 01-julho-1992.

Dr. Jayme Adamo Júnior - Rua Conselheiro Furtado 1155 apto. 63 - 01511-001 São Paulo SP - Brasil. 
lhante que năo mora em săo Paulo, năo senđo possivel examiná-lo; nega consaguinidade. Exame clínico geral normal. Exame neurológico - Consciente, orientado, movimentos córeoatetóicos (pescoco, face e membros) com maior frequencia a direita; postura distónica das mäos, pescoco e face; pé direito equino-varo; reflexos profundos exaltados globalmente; reflexo cutâneo-plantar năo obtiđao bilateralmente; tônus muscular aumentado com sinal da roda denteada no punho direito (espasticidade e rigidez plástica); fundo de olho obtida (lampada de fenda) não mostrou alterações; pesquisa do lanel de Kayser-Fleisher ausente. Exames complementares - Enzimas hepáticas normais, ceruloplasmina normal, teste anti-HIV negativo. Liquido cefalorraquidiano normal. Eletromiografia: reflexo H (obtido através do nervo tibial) com amplitude aumentada. Tomografia computadorizada de crânio normal (Fig. 1A). Pela evolução
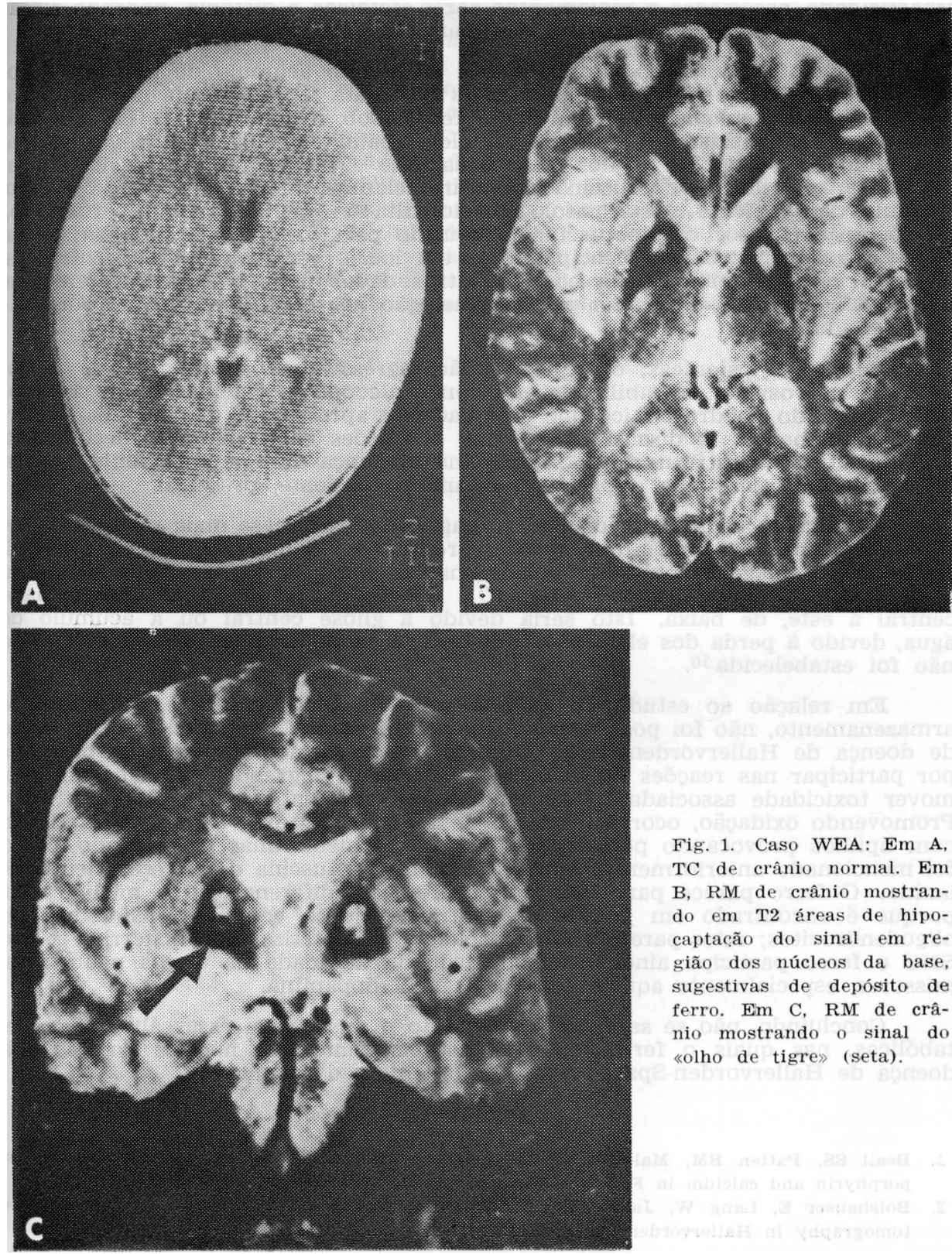

Fig. 1. Caso WEA: Em A, TC de crânio normal. Em B, RM de crânio mostrando em T2 áreas de hipocaptação do sinal em região dos núcleos da base, sugestivas de depósito de ferro. Em C, RM de crânio mostrando o sinal do «olho de tigre» (seta). 
e pelo quadro clínico foi feita la hipótese diagnóstica de doença de Hallervorden-Spatz; por isso foi solicitada RM do crânio, que confirmou a hipótese (Fíg. 1B). Existem poucos dados na literatura sobre o tratamento medicamentoso; este paciente vem recebendo biperideno e benzodiazepínico, com boa melhora no inicio do tratamento; porém, o quadro clínico continua progredindo com piora da agressividade, da depressão e da dísfagia, tendo sido tentadosi outroe medicamentos sem sucesso; com o esquema terapêtico mencionado é que foi obtido melhor resultado.

\section{COMENTARIOS}

A doença de Hallervorden-Spatz caracteriza-se por progressiva rigidez $\theta$ espasticidade, associadas a movimentos córeo-atetóicos e distonia, podendo ainda haver quadro demencial associado e aumento da pigmentação da retina 2 .

Em estudos anátomo-patológicos foi registrado acúmulo de pigmento rico em ferro, depositado no globo pálido, pars reticulata da substância negra, núcleo rubro e corpo geniculado medial 11. O ferro, sob forma férrica $(\mathrm{Fe}+3)$, está presente em quantidades excessivas no globo pálido e substância negra e está diminuído em outras partes dos núcleos da base 5 . O ferro parece catalisar uma pseudo-peroxidação da lipofuscina em neuromelanina, a qual é responsável pelo acúmulo de pigmento nesta patologia. Acredita-se que um lipídio peroxidado, que é um precursor da lipofuscina, produzido pela oxidação da mielina, possa ser tóxico aos neurônios, principalmente em locais com grandes concentrações de ferro como os núcleos da base 5 . Outros dados de literatura sugerem que exista deficiência de dopamina pela degeneração neuronal, comprovada por análise histoquímica 5 .

Alteraçōes psíquicas, como depressão, agressividade, mudanças de personalidade, nervosismo, instabilidade emocional, alcoolismo e tentativa de suicidio, fazem parte do quadro clínico que este paciente apresenta e que vem se exacerbando atualmente. discutível se estas alterações são realmente decorrência de uma alteração estrutural, ou sejam uma mudança de comportamento reativa às alterações físicas que o paciente, estando consciente, presencia 6 .

Atualmente, com o uso da RM, o diagnóstico tornou-se mais seguro. A presença de ferro em grandes quantidades produz em T2 uma diminuição de sinal, formando a imagem conhecida como sinal do olho de tigre, que aparece na região dos núcleos da base 12 (Fig. 1C). Existe um sinal de alta intensidade central a este, de baixa. Isto seria devido a gliose central ou a acúmulo de água, devido à perda dos elementos celulares; a exata natureza deste sinal, ainda não foi estabelecida 10.

Em relação ao estudo do metabolismo do ferro, absorção, transporte $e$ armazenamento, não foi possível até o momento excluir ou firmar o diagnóstico de doença de Hallervorden-Spatz. O ferro possui grande importância biológica por participar nas reações de oxidação; por isso tem grande facilidade em promover toxicidade associada a seu acúmulo no sistema nervoso central (SNC). Promovendo oxidação, ocorre liberação de radicais hidroxila $\left(\mathrm{OH}^{-}\right)$, que reagem com lipídios provocando peroxidação destes nas membranas e formando, como foi mencionado anteriormente, compostos como lipofuscina e, às vezes, neuromelanina. O ferro parece participar da proliferação, diferenciação e mielinização, porque é encontrado em grande concentração, junto com a transferrina, em oligodendrócitos; estes parecem ter capacidade de sintetizar a transferrina 14. No SNC o ferro participa ainda na modulação da atividade de certos neurotrans. missores, especialmente aqueles que utilizam a dopamina.

Concluindo, não se sabe até o momento o que leva a essas alterações metabólicas, nas quais o ferro participa especialmente nos núcleos da base na doença de Hallervorden-Spatz, sendo ainda objeto de estudos.

\section{REFERENCIAS}

2. Beall SS, Patten BM, Mallette L, Jankpvoc J. Abnormal systemic metabolism of iron, porphyrin and calcium in Fahr's syndrome. Ann Neurol 1989, 26:569-575.

2. Bolshauser E, Lang W, Janzer R, Briner J, Spiess H, Klelhues P. Isler W. Computed tomography in Hallervorden-Spatz disease. Neuropediatrics 1987, 18:81-83. 
3. Bresolin AU, Pascuzzi L, Melaragno RFo, Pecora M, Souza Dias JC. Hallervorden-Spatz syndrome-infantile neuroaxonal dystrophy complex: case report. Arq Neuro-Psiquiat 1988, 46:69-72.

4. Dooling EC, Richardson EPJr, Davis KR. Computed tomography in Hallervorden-Spatz disease. Neurology 1980, 30:1128-1130.

5. Jankovic J, Kirkpiatrick JB, Blomquist KA, Langlais PJ, Bird ED. Late onset Hallervorden-Spatz disease presenting as familial parkinsonism. Neurology 1985, 35:227-234.

6. Morphy MA, Feldman JA, Kilburn G. Hallervorden-Spatz disease, J Clin Psqchiatry 1989 , $50: 66-68$.

7. Mutoh $\mathbf{K}$, Okuno $T$, Ito $M$, Nakano $S$, Mikawa $H$, Fujisiawa I, Asato R. MRI of group I case of Hallervorden-Spatz disease. J Comput Assist Tomogr 1988, 78:851-853.

8. Roy RC, McLain S, Wise A, Shaffner LS. Anesthesic management of a patient with Hallervorden-Spatz disease. Anesthesiology 1983, 58:382-384.

9. Russo LSJir. Focal dystonia and lacunar infarction of the basal ganglia. Arch Neurol 1983, 40:61-62.

10. Rutledge JN, Hilal SK, Silver AJ, Defendini R, Fahn S. Study of movement disorders and brain iron by MR. Am J Neuroradiol 1987, 8:397-411.

11. Schaffert DA, Johnsen SD, Johnson PC, Drayer BP. Magnetic resonance imaging in pathologically proven Hallervorden-Spatz disease. Neurology 1989, 39:440-442.

12. Sethi KD, Adams RJ, Loring DW, El Gammal T. Hallervorden-Spatz syndrome: clinical and magnetic resonance imaging correlations Ann Neurol 1988, 24:692-694.

13. Swaiman KF, Smith SA, Trock GL, Siddiqui AR. Sea blue histiocytes, lymphocytic cytosomes, movement disorder and Fe-uptake in basal ganglia: Hallervorden-Spatz disease or ceroid storage with abnormal isotope scan? Neurology 1983, 33:301-305.

14. Swaiman KF. Hallervorden-Spatz syndrome and brain iron metabolism. Arch Neurol 1991, 48:1285-1293. 